\title{
Comparison of 3 Devices for Automated Infusion of Positron-Emitting Radiotracers
}

\author{
Noriaki Miyaji*1, Kenta Miwa*2, Kei Wagatsuma ${ }^{3}$, Taisuke Murata ${ }^{4}$, Takuro Umeda ${ }^{1}$, Takashi Terauchi ${ }^{1}$, \\ and Mitsuru Koizumi ${ }^{1}$ \\ ${ }^{1}$ Department of Nuclear Medicine, Cancer Institute Hospital of Japanese Foundation for Cancer Research, Tokyo, Japan; ${ }^{2}$ Department \\ of Radiological Sciences, School of Health Sciences, International University of Health and Welfare, Tochigi, Japan; ${ }^{3}$ Research \\ Team for Neuroimaging, Tokyo Metropolitan Institute of Gerontology, Tokyo, Japan; and ${ }^{4}$ Department of Radiology, Chiba University \\ Hospital, Chiba, Japan
}

The administration accuracy and precision of an automated infusion device for positron-emitting radiotracers are directly associated with bias and variance in the SUVs of ${ }^{18} \mathrm{~F}-\mathrm{FDG}$ PET/CT. Therefore, the accuracy of such devices must be confirmed and calibrated at locations in which they are used. The present study aimed to validate the administration accuracy of 3 automated infusion devices for quantitative PET assessment. Methods: Temporal variations as well as variations in radioactive concentrations and dispensed volumes of ${ }^{18} \mathrm{~F}-\mathrm{FDG}$ were determined for the M-130, Al-300, and UG-05 automated infusion devices. The total-test dispensed volumes were 25, 20, and $18.5 \mathrm{~mL}$, respectively. A reference value was generated by measuring amounts of radioactivity using a standard dose calibrator. Administration accuracy was validated according to the criteria of the Japanese Society of Nuclear Medicine. Results: The temporal variation in the M-130 and UG-05 for a specified $185 \mathrm{MBq}$ was relatively stable, in the range of $-1.60 \%-0.92 \%$ and $1.16 \%-5.35 \%$, respectively, whereas that in the Al-300 was $-0.55 \%-8.68 \%$. For the M-130 and UG-05 devices, the difference between measured and reference value was in the range of $-5 \%-5 \%$. The values measured by the $\mathrm{Al}-300$ deviated from the reference values by a maximum of $30 \%$, which depends on radioactive concentration and dispensed volume of ${ }^{18} \mathrm{~F}-\mathrm{FDG}$. Conclusion: The administration accuracy of the Al-300 varied considerably under different conditions, but a software update might somewhat improve this. Our findings indicate that dispensed volumes of ${ }^{18} \mathrm{~F}-\mathrm{FDG}$ should be carefully considered when the radioactive concentration is high. Administration accuracy should be regularly confirmed at each location to maintain the quality of quantitative PET assessment. The present study provides useful information about how to confirm the administration accuracy of automated infusion devices.

Key Words: positron emission tomography (PET); administration accuracy; automated infusion device; quantitative PET; dose calibrator; radiation exposure

J Nucl Med Technol 2017; 45:91-95

DOI: $10.2967 /$ jnmt.116.188243

Received Dec. 8, 2016; revision accepted Feb. 28, 2017.

For correspondence or reprints contact: Kenta Miwa, Department of Radiological Sciences, School of Health Sciences, International University of Health and Welfare, 2600-1 Kitakanemaru, Ohtawara, Tochigi 324-8501, Japan

E-mail: kenta5710@gmail.com

${ }^{*}$ Contributed equally to this work.

Published online Mar. 9, 2017.

COPYRIGHT (c) 2017 by the Society of Nuclear Medicine and Molecular Imaging.
$\mathbf{P}$ tant for the initial staging, differential diagnosis, treatment response, and prognostic prediction of various malignancies (1-3). The incidence of ${ }^{18} \mathrm{~F}-\mathrm{FDG}$ PET/CT evaluations is increasing worldwide, and thus exposing nuclear medicine workers to increasing amounts radiation during increasingly frequent administrations of ${ }^{18} \mathrm{~F}-\mathrm{FDG}$ to patients is problematic (4-6). Automated infusion devices for positron-emitting radiotracers have recently been developed to reduce radiation exposure. Schleipman et al. showed that an automated infusion device resulted in an estimated 10-fold reduction in whole-body and extremity exposure among nuclear medicine workers (7). Automated infusion devices have been installed in most (87.1\%) Japanese PET facilities (8), but their quantitative performance and longitudinal accuracy have not yet been evaluated.

The accurate and reproducible administration of ${ }^{18} \mathrm{~F}$ FDG is directly associated with bias and variance in SUV because the administration accuracy to the patient is used directly in the SUV equation (9). Boellaard reported that variations of factors affecting ${ }^{18} \mathrm{~F}-\mathrm{FDG}$ PET quantification ranged from $0 \%$ to $50 \%$ or more depending on the quality of administration (10). Regular quality control of the administration accuracy and precision of automated infusion devices is essential to maintain their performance. Accuracy may vary within $3 \%$ between automated infusion devices and dose calibrators according to the guidelines for ${ }^{18}$ F-FDG imaging of the European Association of Nuclear Medicine (EANM) (11). That is, the actual amount of administered radioactivity may not deviate by more than $3 \%$ from that indicated by automated infusion devices.

Lecchi et al. recently compared the accuracy of ${ }^{18} \mathrm{~F}-\mathrm{FDG}$ radioactivity administered by the Intego automated infusion device (MEDRAD Inc.) with that administered using a manual dose-delivery system (12). Although they found that variation in the accuracy of the ${ }^{18} \mathrm{~F}-\mathrm{FDG}$ radioactivity administered by the Intego was within $3 \%$ (12), the quantitative accuracy of a wide range of radioactive concentrations and dispensed volumes was not clear. The administration accuracy of automated infusion devices depends on the radioactive ${ }^{18} \mathrm{~F}-\mathrm{FDG}$ 


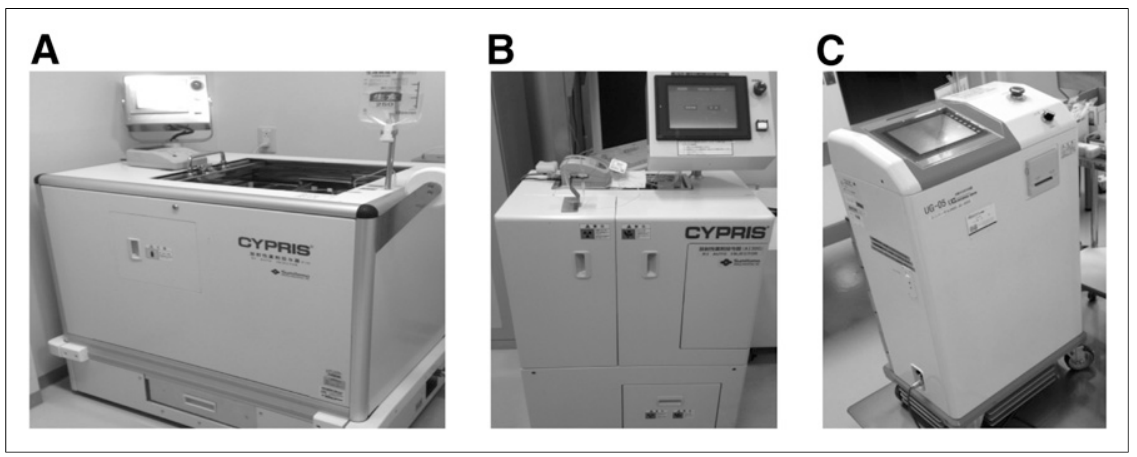

GURE 1. Manufactured automated infusion devices evaluated in this study: M-130 (A), Al-300 (B), and UG-05 (C).

motor-driven 20-mL syringe. Table 1 shows the main technical features of the automated infusion devices compared in this study. If the dispensed volume of ${ }^{18} \mathrm{~F}-\mathrm{FDG}$ solution is greater than $0.25 \mathrm{~mL}$ for the M-130 and $\mathrm{AI}-300$ and $0.3 \mathrm{~mL}$ for the UG-05, then the manufacturers can guarantee the administration accuracy of each device.

\section{Validation of Administration Accuracy}

Preparation of Measurements. We measured amounts of radioactivity using CRC-25PET and CRC-712 external dose calibrators (Capintec Inc.) to validate the administration accuracy of the automated infusion devices. These dose calibrators have been standardized using the National Institute of Standards and

\section{Device Operational Characteristics of Automated Infusion Devices}

We compared the M-130, AI-300 (both Sumitomo Heavy Industries Ltd.), and UG-05 (Universal Giken Co. Ltd.) automated infusion devices (Fig. 1). Figure 2 shows their operational characteristics. The scheme of measurement and administration process of each device is roughly performed by 4 steps. For the first step, radioactive concentration in a multidose vial is automatically measured by each sensor or scintillator. In the second step, the requested activity through a touchscreen computer interface is automatically withdrawn from the multidose vial by a motor-driven syringe. In the third step, the accuracy of the requested activity in each syringe is reconfirmed by each sensor or scintillator. In the final step, to flush the extracted request, ${ }^{18} \mathrm{~F}$-FDG with saline is injected into patient via 3-way cock.

The administration procedure of the M-130 consists of basic processes. The radioactive concentration in a multidose vial is automatically measured by the thallium-activated cesium iodide $(\mathrm{CsI}(\mathrm{Tl}))$ scintillator, and a motor-driven $10-\mathrm{mL}$ syringe withdraws a specific amount of radioactivity from the vial determined through a touchscreen computer interface. The amount of radioactivity in the syringe is reconfirmed by the plastic scintillator. Finally, a solution containing ${ }^{18} \mathrm{~F}-\mathrm{FDG}$ and physiologic saline for flushing and washing is injected into patients via a 3-way stopcock. The other devices have broadly similar device operational characteristics that differ slightly according to each integrated measurement mechanism. The radioactive concentration in multidose vials is automatically measured by the semiconductor sensor in the AI-300, and that in 5-mL syringes is adjusted by 2 semiconductor sensors that are adjacent to the syringe. The radioactive concentration in multidose vials is also automatically measured in the UG-05. A specified amount of radioactivity withdrawn from a $5-\mathrm{mL}$ syringe into a $10-\mathrm{mL}$ vial is confirmed by the semiconductor sensor, and then the specified amount is withdrawn from the $10-\mathrm{mL}$ vial using a

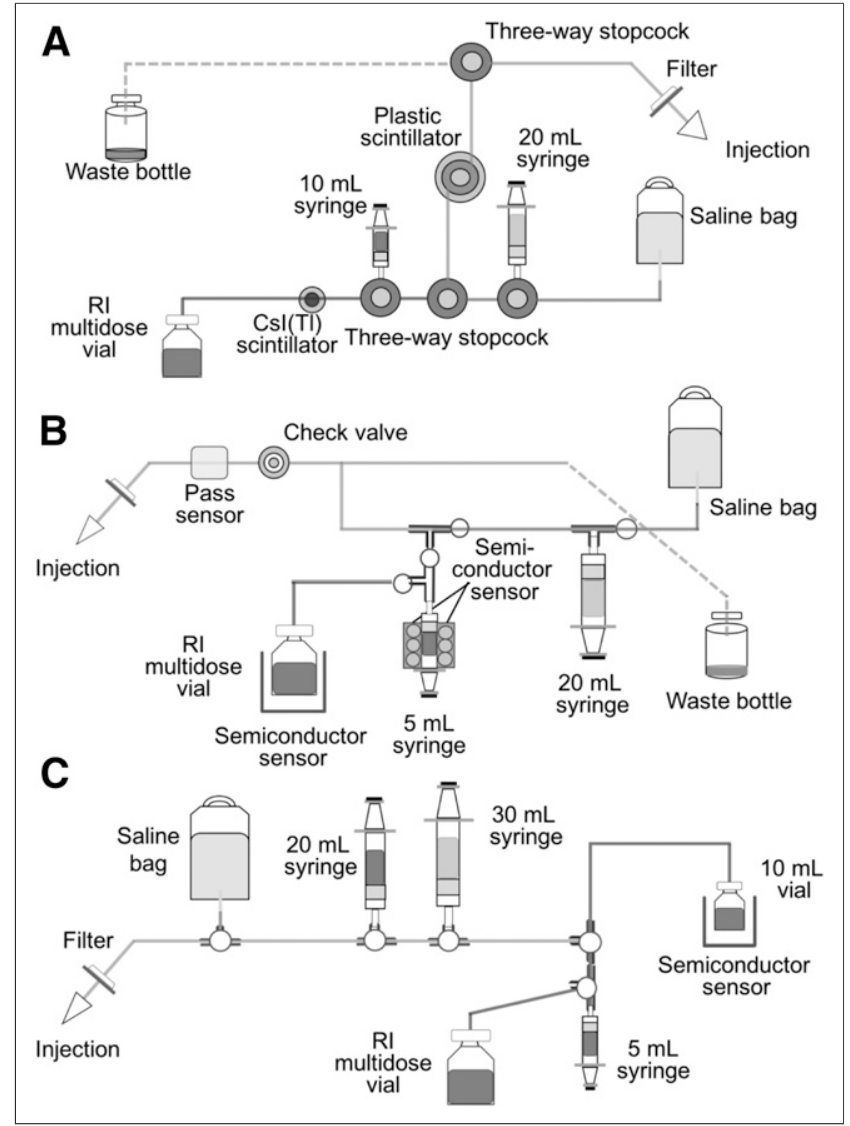

FIGURE 2. Scheme of measurement and administration process of each device: M-130 (A), Al-300 (B), and UG-05 (C). 


\begin{tabular}{|c|c|c|c|}
\hline \multirow[b]{2}{*}{ Feature } & \multicolumn{3}{|c|}{ Automated infusion device } \\
\hline & $\mathrm{M}-130$ & Al-300 & UG-05 \\
\hline Maximum radioactivity of multidose vial (MBq) & 18,500 & 37,000 & 37,000 \\
\hline Maximum radioactive concentration of multidose vial $(\mathrm{MBq} / \mathrm{mL})$ & 1,500 & 1,500 & 1,200 \\
\hline Range of net administration (MBq) & $100-740$ & $50-740$ & -740 \\
\hline Measurement accuracy of radioactive concentration (\%) & \pm 10 & \pm 5 & \pm 5 \\
\hline Measurement accuracy of radioactivity (\%) & \pm 10 & \pm 5 & \pm 5 \\
\hline Administration accuracy (\%) & \pm 10 & \pm 5 & \pm 5 \\
\hline Variation of volume & Constancy & Variability & Constancy \\
\hline Measurement sample & Tube & Syringe & Vial \\
\hline
\end{tabular}

Technology-traceable ${ }^{68} \mathrm{Ge} /{ }^{68} \mathrm{Ga}$ standard sources as ${ }^{18} \mathrm{~F}$ surrogates (14). We set the same time on dose calibrator and each device because the quantitative value of ${ }^{18} \mathrm{~F}$ changes by about $0.6 \%$ per minute due to differences in time between dose calibration and automated infusion devices. The external dose calibrator and all internal detectors of the automated infusion devices were directly cross-calibrated using ${ }^{18} \mathrm{~F}$. We also confirmed the total administered volume using the LA230S (Sartorius Corp.) electronic balance as real volume.

Measurement of Administration Accuracy. We validated administration accuracy using the confirmation procedure of the Japanese Society of Nuclear Medicine, which is based on the guidelines for ${ }^{18} \mathrm{~F}-\mathrm{FDG}$ imaging of the EANM (11). The differences of measured value in internal detector of each device and external dose calibrator were confirmed. We determined temporal variations, variations in radioactive concentrations, and dispensed volumes of ${ }^{18} \mathrm{~F}-\mathrm{FDG}$ for the AI-300, M-130, and UG-05. The total volumes of physiologic saline containing ${ }^{18} \mathrm{~F}-\mathrm{FDG}$ in sealed $30-\mathrm{mL}$ vials for the M-130, AI-300, and UG-05 were 25, 20, and $18.5 \mathrm{~mL}$, respectively. A reference value comprised a specific amount of radioactivity in a sealed vial measured using the external dose calibrator. The average of 5 measured values was defined as the reference value with which all radioactivity values determined by the automated infusion devices were compared. Temporal variations in specified amounts of 50 and $185 \mathrm{MBq}$ of radioactivity were measured 6 times on different days. The results of sample 6 for the M-130 were excluded because they did not fulfil the criteria. Variations in radioactive concentrations and dispensed volumes of ${ }^{18} \mathrm{~F}$-FDG were also confirmed with $100-1,000 \mathrm{MBq} / \mathrm{mL}$ and $0.15-3.5 \mathrm{~mL}$, respectively. Radioactive concentrations were calculated by dividing the actual administered volume by the desired amount of administered radioactivity.

The amount of radioactivity measured by automated infusion devices must be corrected to the value at the time of the external measurement using a dose calibrator to account for radioactive decay. The administration error of the automated infusion device was as follows:

$$
\begin{aligned}
\text { Administration error }(\%)= & \left\{\frac{A_{a}}{A_{m}} \times \exp \left(-\frac{T_{m}}{109.8} \times \ln (2)\right)-1\right\} \\
& \times 100
\end{aligned}
$$

where $A_{a}$ is the amount of radioactivity recorded by automated infusion device, $A_{m}$ is the amount of radioactivity measured using the dose calibrator, $T_{m}$ is the difference between the time of administration by the automated device and the time when the amount of radioactivity was measured by the dose calibrator (min), and 109.8 is the half-life of ${ }^{18} \mathrm{~F}$ ( $\left.\mathrm{min}\right)$.

The manufacturer released updated software for the AI-300 in 2015 to improve administration accuracy. We compared administration error before and after the software release.

\section{RESULTS}

Table 2 summarizes temporal variations in administration error on different days. The mean administration accuracy of the AI-300 was $7.43 \%$ (range, $1.52 \%-14.42 \%$ ) and 5\% (range, $-0.55 \%-8.68 \%$ ) with specified amounts of 50 and $185 \mathrm{MBq}$ of radioactivity, respectively. The variability was appreciably higher than that of the others devices. The mean administration error of the M-130 for 50 and $185 \mathrm{MBq}$ of radioactivity was stable within $-2.86 \%$ (range, -3.79 to $-1.59 \%$ ) and $-0.80 \%$ (range, $-1.60 \%-0.92 \%$ ), respectively, whereas that of the UG-05 was $4.26 \%$ (range, $-0.13 \%-6.37 \%$ ) and $3.48 \%$ (range, $1.16 \%-5.35 \%$ ), respectively.

Figure 3 shows the administration error of the devices as a function of the radioactive concentration and the dispensed volume of ${ }^{18} \mathrm{~F}$-FDG. The administration error of the AI-300 reached a maximum of $30 \%$ at a high radioactive concentration of $700 \mathrm{MBq} / \mathrm{mL}$, and bias appeared proportional to the radioactive concentration (Fig. 3A). The M-130 and UG-05 were relatively stable at $-5 \%$ and $5 \%$, respectively. On the other hand, bias to the dispensed volume of ${ }^{18} \mathrm{~F}-\mathrm{FDG}$ was inversely proportional in the AI-300 (Fig. 3B). The percentage deviation was large when the dispensed volume was less than $0.5 \mathrm{~mL}$, reaching a maximum of $30 \%$. The M-130 and UG-05 achieved stability to the dispensed volume of ${ }^{18} \mathrm{~F}-\mathrm{FDG}$ in the range of $-5 \%-5 \%$.

Figure 4 shows the administration error of the AI-300 before and after the software update. The administration error improved to within $5 \%$ after the update, but was worse by $-10 \%$ when the dispensed volume was less than a $0.2-\mathrm{mL}$ volume; this is not covered by a warranty.

\section{DISCUSSION}

Although manufactured automated devices for infusing positron-emitting radiotracers have common features, many points differ and their administration accuracy and precision under various administration conditions are not 
TABLE 2

Comparison of Accuracy of Administered ${ }^{18}$ F-FDG Radioactivity Between Automated Infusion Device and External Dose Calibrator

\begin{tabular}{|c|c|c|c|c|c|c|c|c|c|c|c|}
\hline Devices & Sample & $\begin{array}{c}\text { Radioactive } \\
\text { concentration } \\
(\mathrm{MBq} / \mathrm{mL})\end{array}$ & $\begin{array}{c}\text { Dispensed } \\
\text { volume of } \\
{ }^{18} \mathrm{~F}-\mathrm{FDG} \\
(\mathrm{mL})\end{array}$ & $\begin{array}{l}\text { Radioactivity } \\
\text { recorded by } \\
\text { device (MBq) }\end{array}$ & $\begin{array}{l}\text { External } \\
\text { measurement } \\
(\mathrm{MBq})\end{array}$ & $\begin{array}{c}\text { Administration } \\
\text { accuracy (\%) }\end{array}$ & $\begin{array}{c}\text { Radioactive } \\
\text { concentration } \\
(\mathrm{MBq} / \mathrm{mL})\end{array}$ & $\begin{array}{c}\text { Dispensed } \\
\text { volume of } \\
{ }^{18} \mathrm{~F}-\mathrm{FDG} \\
(\mathrm{mL})\end{array}$ & $\begin{array}{l}\text { Radioactivity } \\
\text { recorded by } \\
\text { devise (MBq) }\end{array}$ & $\begin{array}{c}\text { External } \\
\text { measurement } \\
(\mathrm{MBq})\end{array}$ & $\begin{array}{c}\text { Administration } \\
\text { accuracy (\%) }\end{array}$ \\
\hline \multirow[t]{6}{*}{$M-130$} & 1 & 294.12 & 0.17 & 48.42 & 50.33 & -3.79 & 225.94 & 0.84 & 189.79 & 192.89 & -1.60 \\
\hline & 2 & 121.95 & 0.41 & 48.40 & 49.76 & -2.72 & 127.32 & 1.40 & 178.25 & 179.58 & -0.74 \\
\hline & 3 & 200.00 & 0.25 & 49.26 & 50.05 & -1.59 & 202.31 & 0.87 & 176.01 & 178.43 & -1.36 \\
\hline & 4 & 147.06 & 0.34 & 48.52 & 49.84 & -2.66 & 152.30 & 1.17 & 178.20 & 180.36 & -1.20 \\
\hline & 5 & 166.67 & 0.30 & 48.19 & 49.97 & -3.56 & 167.53 & 1.04 & 174.23 & 172.64 & 0.92 \\
\hline & 6 & 142.86 & 0.35 & 24.07 & 24.42 & -1.42 & 59.61 & 1.54 & 91.80 & 94.27 & -2.63 \\
\hline \multirow[t]{6}{*}{ Al-300 } & 1 & 104.17 & 0.48 & 48.87 & 47.87 & 2.09 & 118.80 & 1.42 & 168.70 & 169.63 & -0.55 \\
\hline & 2 & 131.58 & 0.38 & 50.09 & 49.34 & 1.52 & 165.57 & 1.12 & 185.44 & 170.62 & 8.68 \\
\hline & 3 & 147.06 & 0.34 & 50.61 & 44.54 & 13.64 & 178.59 & 1.04 & 185.73 & 175.66 & 5.73 \\
\hline & 4 & 131.58 & 0.38 & 52.01 & 46.88 & 10.94 & 161.71 & 1.14 & 184.35 & 173.83 & 6.05 \\
\hline & 5 & 151.52 & 0.33 & 49.01 & 42.83 & 14.42 & 177.28 & 1.05 & 186.14 & 171.39 & 8.61 \\
\hline & 6 & 161.29 & 0.31 & 48.93 & 47.98 & 1.99 & 183.62 & 1.01 & 185.46 & 182.75 & 1.48 \\
\hline \multirow[t]{6}{*}{ UG-05 } & 1 & 90.79 & 0.60 & 54.48 & 51.27 & 6.26 & 92.04 & 2.01 & 180.23 & 174.92 & 3.04 \\
\hline & 2 & 154.73 & 0.34 & 52.61 & 50.16 & 4.87 & 181.37 & 1.02 & 147.90 & 141.92 & 4.21 \\
\hline & 3 & 205.09 & 0.26 & 53.32 & 52.14 & 2.27 & 205.56 & 0.90 & 185.01 & 175.61 & 5.35 \\
\hline & 4 & 206.85 & 0.26 & 53.78 & 50.56 & 6.37 & 179.61 & 1.03 & 179.35 & 177.29 & 1.16 \\
\hline & 5 & 224.45 & 0.25 & 56.11 & 52.98 & 5.92 & 212.64 & 0.87 & 182.29 & 173.10 & 5.31 \\
\hline & 6 & 108.58 & 0.39 & 42.34 & 42.40 & -0.13 & 411.11 & 0.45 & 191.05 & 187.63 & 1.83 \\
\hline
\end{tabular}

understood in detail. We validated the administration accuracy of 3 automated infusion devices. The results indicated that the radioactive concentration and dispensed volume of ${ }^{18} \mathrm{~F}$-FDG affected the administration accuracy of the devices to various degrees and that the AI-300 was unstable.

Values were measured under various conditions on different days to exclude factors such as the impact of operator error and hardware. Temporal variations in desired amounts of radioactivity adversely affected all automated infusion devices more at 50 than at $185 \mathrm{MBq}$. The administration accuracy at $50 \mathrm{MBq}$ deviated from the accuracy of each device guaranteed by manufacturers and the EANM criteria (11). We consider that the measurement accuracy of the devices is limited at lower levels of ${ }^{18} \mathrm{~F}$ FDG radioactivity and thus the administration accuracy might be reduced. The degree of temporal variation differed depending on the device. The administration error of $185 \mathrm{MBq}$ was stable for the M-130 and UG-05 at $-0.80 \%$ (range, $-1.60 \%-0.92 \%$ ) and $3.48 \%$ (range, $1.16 \%-5.35 \%$ ), respectively, which was closer to the accuracy in a previous study of the Intego system that met the EANM criteria (12).
These results indicated that the M-130 and UG-05 devices were stable and offered highly reproducible administration accuracy.

The desired volume of ${ }^{18} \mathrm{~F}-\mathrm{FDG}$ dispensed by each device declines as a function of a higher radioactive concentration when extracted from multidose vials (13). Therefore, a dispensed volume below $0.5 \mathrm{~mL}$ conspicuously worsened the administration error of the AI-300, which was already overestimated compared with the other devices. The device operational characteristics of the AI-300 is a key factor in the overestimation. The manufacturer of the AI-300 has publicly announced that this was primarily caused by the ${ }^{18} \mathrm{~F}-\mathrm{FDG}$ solution and physiologic saline adhering to the boundary of the 3-way stopcock. Systematic error can be explained by the ${ }^{18}$ F-FDG solution mixing with saline at the boundary of 3-way stopcock over time, or a small amount of ${ }^{18}$ F-FDG detaching from a stock ${ }^{18} \mathrm{~F}-\mathrm{FDG}$ during flushing with saline. Thus, we considered that an overestimated amount of radioactivity administered by the AI-300 as a function of a smaller dispensed volume of ${ }^{18}$ F-FDG would increase uncertainty relative to the total administered volume.
FIGURE 3. Variations in radioactive concentrations $(\mathrm{A})$ and dispensed volumes (B). $\circ=\mathrm{Al}-300 ; \bullet=\mathrm{M}-130 ; \times=\mathrm{UG}-05$.

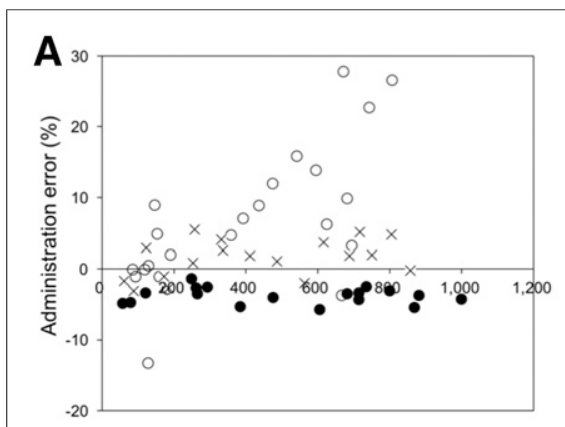

Radioactive concentration $(\mathrm{MBq} / \mathrm{mL})$

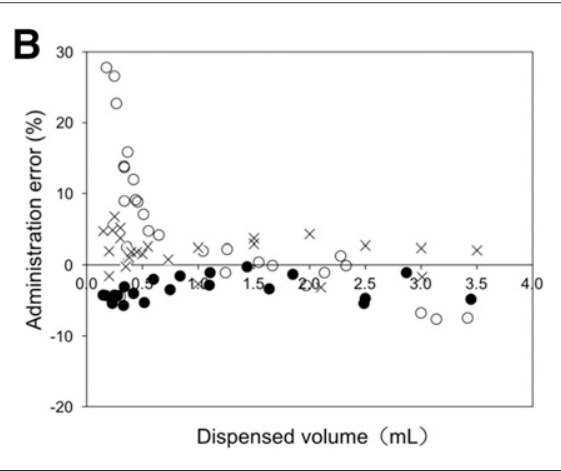




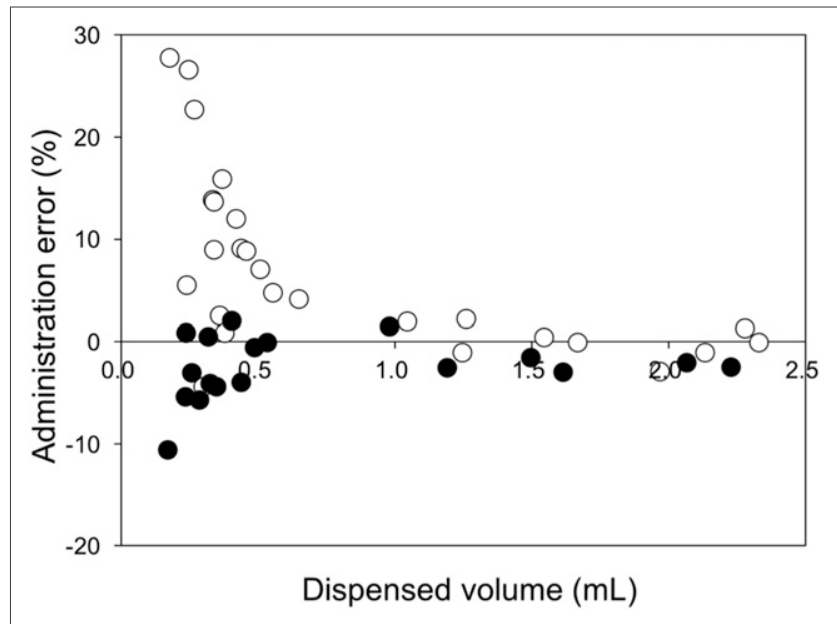

FIGURE 4. Administration accuracy of Al-300 before and after software update. $\bigcirc=$ original; $\bullet=$ update.

The manufacturer of the AI-300 updated the software to suppress overestimation. It automatically adjusts the dispensed volume of ${ }^{18} \mathrm{~F}$-FDG to improve the accuracy of dispensed volumes below $0.5 \mathrm{~mL}$. The dispensed volume of radioactivity after updating the software was stable within 5\% (Fig. 4). Systematic error in the AI-300 should therefore have a much smaller effect after the software update. However, because correction by software cannot improve the integrated device operational characteristics, systematic error in the AI-300 cannot be completely resolved in this manner. A new device with refinements is currently under development to replace the AI-300.

Although the 3 automated infusion devices barely met the EANM criterion of $\pm 3 \%$ (11), we confirmed that the administration error of the M-130, AI-300, and UG-05 was within the assurance range stated by the manufacturers $( \pm 10 \%, \pm 5 \%$ and $\pm 5 \%$, respectively). A method of managing the administration accuracy of automated infusion devices has not yet been defined, but the present study provides useful information about how to confirm it. We suggest that consistent administration accuracy can be achieved in the following 3 ways. The tendency of error inherent in each device at each location should be confirmed. Users and manufacturers should regularly correct, maintain, and implement quality control measures for automated infusion devices by rigorous cross-calibration between the dose calibrator and the integrated detectors within each device. The optimal radioactive concentration should be determined, and then the dispended volume of ${ }^{18} \mathrm{~F}-\mathrm{FDG}$ should be adjusted with the operational aspects of clinical practice taken into consideration. We do not believe that these ways are sufficient to resolve all issues associated with the administration accuracy of automated infusion devices and will require further investigation.

This study is limited by our comparison of 3 automated infusion devices and focus on their administration accuracy. Further study is required to evaluate the radiation protection ability and operational aspects of automated infusion devices $(4,7,12,13)$.

\section{CONCLUSION}

The administration accuracy of the AI- 300 was unstable under various conditions, but a software update provided some improvement. Our results indicate that the dispensed volumes at high radioactive concentrations is important. The elimination of administration error from high radioactive concentrations makes it possible to reflect mainly the level of guideline required for the intended clinical use of quantitative PET. In addition, administration accuracy should be regularly confirmed at each location to maintain the quality of quantitative PET assessment. The present study provides useful information about how to confirm the administration accuracy of automated infusion devices.

\section{DISCLOSURE}

No potential conflict of interest relevant to this article was reported.

\section{REFERENCES}

1. Fischer B, Lassen U, Mortensen J, et al. Preoperative staging of lung cancer with combined PETCT. $N$ Engl J Med. 2009;361:32-39.

2. Jiang $\mathrm{C}$, Zhang $\mathrm{X}$, Jiang $\mathrm{M}$, et al. Assessment of the prognostic capacity of pretreatment, interim, and post-therapy ${ }^{18} \mathrm{~F}-\mathrm{FDG} \mathrm{PET} / \mathrm{CT}$ in extranodal natural killer/T-cell lymphoma, nasal type. Ann Nucl Med. 2015;29:442-451.

3. Ito R, Iwano S, Kishimoto M, Ito S, Kato K, Naganawa S. Correlation between FDG-PET/CT findings and solid type non-small cell cancer prognostic factors: are there differences between adenocarcinoma and squamous cell carcinoma? Ann Nucl Med. 2015;29:897-905.

4. Sánchez RM, Vano E, Fernández JM, Ginjaume M, Carreras JL. Evaluation of an automated FDG dose infuser to PET-CT patients. Radiat Prot Dosimetry. 2015;165: $457-460$.

5. Antic V, Ciraj-Bjelac O, Stankovic J, Arandjic D, Todorovic N, Lucic S. Radiation exposure to nuclear medicine staff involved in PET/CT practice in Serbia. Radiat Prot Dosimetry. 2014;162:577-585.

6. Guillet B, Quentin P, Waultier S, Bourrelly M, Pisano P, Mundler O. Technologist radiation exposure in routine clinical practice with ${ }^{18} \mathrm{~F}$-FDG PET. J Nucl Med Technol. 2005;33:175-179.

7. Schleipman AR, Gerbaudo VH. Occupational radiation dosimetry assessment using an automated infusion device for positron-emitting radiotracers. $\mathrm{J} \mathrm{Nucl}$ Med Technol. 2012;40:244-248.

8. Honda N, Fukukita H, Iida K, et al. Report on present status of quality control and assurance of PET in Japan based on questionnaire survey. Radioisotopes. 2012;61:555-569.

9. Boellaard R, Oyen WJ, Hoekstra CJ, et al. The Netherlands protocol for standardisation and quantification of FDG whole body PET studies in multi-centre trials. Eur J Nucl Med Mol Imaging. 2008;35:2320-2333.

10. Boellaard R. Standards for PET image acquisition and quantitative data analysis. J Nucl Med. 2009;50(suppl 1):11S-20S.

11. Boellaard R, Delgado-Bolton R, Oyen WJ, et al. FDG PET/CT: EANM procedure guidelines for tumour imaging: version 2.0. Eur J Nucl Med Mol Imaging. 2015;42:328-354.

12. Lecchi M, Lucignani G, Maioli C, Ignelzi G, Del Sole A. Validation of a new protocol for ${ }^{18} \mathrm{~F}-\mathrm{FDG}$ infusion using an automatic combined dispenser and injector system. Eur J Nucl Med Mol Imaging. 2012;39:1720-1729.

13. O'Doherty J, Woods E, Modde L, Mackewn J. Validation of an automated dosedispensing system for ${ }^{18} \mathrm{~F}-\mathrm{FDG}$ administrations and associated reduction in operator extremity dose. Nucl Med Commun. 2014;35:151-159.

14. Zimmerman BE, Cessna JT. Development of a traceable calibration methodology for solid ${ }^{68} \mathrm{Ge} /{ }^{68} \mathrm{Ga}$ sources used as a calibration surrogate for ${ }^{18} \mathrm{~F}$ in radionuclide activity calibrators. $J$ Nucl Med. 2010;51:448-453. 